\title{
Analysis on Application of GIS Technology in Engineering Survey
}

\author{
Yan $\mathrm{LI}^{1, \text { a }}$, Chen ZHANG ${ }^{1, b}$, Weikai ZHANG ${ }^{1, c}$, Shanshan ${ }^{1, \mathrm{~d}}$ ZHAO and Siyan \\ LIU ${ }^{1, \mathrm{e}}$ \\ ${ }^{1}$ Hebei Normal University of Science \& Technology, Qinhuangdao, Hebei, 066004, China \\ aemail:liyan3311@126.com, bemail: fly72010@aliyun.com, 'email: nqwzwk2008@126.com, 'email: \\ susanzhao0809@163.com, email: liusiyan668@sina.cn
}

Keywords: Engineering survey; GIS technology; Modern surveying and mapping technology; Intelligentization; Visualization

\begin{abstract}
Engineering survey, as the basis of engineering construction, is applied throughout all stages of the whole project. However, traditional engineering survey technology cannot satisfy the actual needs of modern engineering any more. Modern Engineering Survey is no longer a narrow concept to serve engineering construction from controlling to mapping. Its service scope and objects have been continuously expanded to numerous fields that are related to geographic spatial data. Geographic Information System (GIS) technology has been widely used in engineering survey due to its advantages of rapidness, accuracy, intelligence and visibility. The GIS technology combines computer technology with geographic spatial distribution data, which provides useful information for management and decision making in various fields. Its application is a technological innovation of engineering survey. Based on a brief introduction of GIS technology, this paper focuses on the four main applications of GIS technology in engineering survey, and the development of modern surveying under the impetus of GIS technology. The paper points out that GIS technology has had extremely important influence on the rapid development of modern construction and created economic benefits for engineering construction.
\end{abstract}

\section{Introduction}

The increase of scientific and technological level and the acceleration of social construction schedule have impelled the rapid development of technical optimization and innovation in engineering construction. Through many stages of development, from Map of China in the Kangxi Reign, the earliest map in the Chinese history, to the comprehensive cartographic information system, the science of surveying and mapping has entered into the information era, during which new theory, new technology and new instrument [1] have appeared in surveying and mapping. Traditional technology of engineering survey can no longer meet the requirements for rapidity and accuracy in modern engineering construction. Therefore, engineering survey must keep pace with the times to continuously renovate the instrument and methods of surveying and mapping. Meanwhile, we need to lay emphasis on comprehensive application of new science and technology in order to move forward from a single discipline to multi-disciplinary intersection.

With the help of computer technology and the knowledge of information and space sciences, modern surveying and mapping technology is mainly a shift, from the traditional one, to the digitization and informationalization of surveying and mapping. With 3S (GPS, RS, GIS) technologies as the core, it is to realize digitized surveying and mapping contents, networked information distribution and popularized surveying and mapping data service [2]. Automated and intelligent modern technology of surveying and mapping has reduced the burden of manual operation, and it has, at the same time, improved surveying accuracy, merged geographic spatial information resources, realized their value-added service and provided multi-scale, multi-aspect and multi-form service to the society. 


\section{Overview}

Geographic information, which is an immense information source of engineering construction, is also the basic data for all types of engineering constructions. Therefore, the collection, management, processing, analysis, updating and utilization of geographic information data are the main tasks of modern surveying and mapping. Geographic Information System (GIS) technology describes all types of objective entities on the globe by way of digital information. Then, with the support of computer and modern information technology, all kinds of geographic information are input, saved, controlled, retrieved, updated, displayed, processed, synthetically analyzed and applied in certain formats according to their spatial distribution and attributes, so they have specific functions for spatial inquiry, analysis and representation. It is a digitized geographic data management system [3]. It can, timely and accurately, provide geographic information for related aspects such as regional analysis, scheme optimization and strategic decision making.

The operation objects of GIS are spatial data, which are provided, in different scales and accuracies, by geodetic survey, engineering survey, aerial photogrammetry and remote sensing technology. Application of GIS technology in surveying is helpful to the management and maintenance of mass data. It can display geographic data in the form of map graphics and generate electronic maps, from which geographic spatial distribution is seen clearly and visually, topographic features are identified explicitly so as to provide data support for various social applications. In the process of cultivating modern surveying and mapping talents, geographic information system course is offered to the students of both surveying and non-surveying specialties. The course is also applied in engineering survey, which is conducive for the students to process and analyze surveying data precisely and quickly, to make electronic maps, to acquire thematic information for various applications and to realize visualized representation of geographic spatial information.

\section{Applications of GIS in Engineering Survey}

A. Intelligent data management and application. The main purpose of topographic survey is to make topographic maps. The method for making topographic maps has been changed, from the traditional plane-table mapping and theodolite mapping, to total station digital mapping, aerial photogrammetry, three-dimensional laser scanning mapping and other digitized mapping methods. With the continuous development of geographic information system, the spatial analysis function of GIS has been continuously enhanced and improved. Thus surveying and mapping of topographic maps is no longer the simple point-line-surface digital mapping. Rather, it is becoming increasingly important to achieve the sharing, distribution and multi-industry utilization of the surveying results [4].

Application of GIS technology in surveying data processing can perform data input intelligently, which could avoid the possible errors t by artificial recognition, calculation and machine plotting in the traditional survey. The enormous capacity of GIS system can achieve management of mass data, which has effectively solved the problems of data management in traditional survey. Moreover, to replace traditional hand-made paper maps, it is convenient to make electronic maps, in which we can flexibly change map display scale and data format, simplify mapping steps, reduce manual operation burden and cost, and increase working efficiency and accuracy [5].

B. Inquiry function of spatial relationship and attribute characteristics. To inquire, locate and measure spatial objects is one of the basic functions of geographic information system. By means of GIS spatial inquiry function, we may find the position and the spatial scale of a surveying object and their attributes, or the corresponding spatial entity according to the record of a certain attribute, thereby conducting corresponding statistical analysis [3]. Generally, there are three major categories of inquiry functions for complicated spatial data: inquiry based on attribute characteristics, for instance, to inquire the points that a certain surveying route passes; inquiry based on spatial relationship, such as the inquiry of control points within a surveying area; inquiry through a spatially extended SQL language, such as the inquiry of the number of roads wider than $20 \mathrm{~m}$ with the 
gradient less than $10 \%$ there are in more than $200 \mathrm{~m}$ from a point. GIS database effectively links spatial information and attribute information together so that the spatial inquiry function becomes more convenient, faster and more efficient, thus the technical level of information inquiry in engineering survey has been improved [6].

C. Scientific spatial analysis function. Spatial analysis is one of the core functions of Geographic Information System. Its greatest advantage lies in the functions to extract, represent and transmit spatial information. The basic function to achieve spatial analysis in GIS includes spatial inquiry, measurement and computation, buffer zone analysis, overlay analysis, path analysis, spatial interpolation, statistical classification analysis, etc. [3].

By using GIS technology, large quantities of survey data can be systematically managed, classified and displayed hierarchically. The spatial index function may, from the database, quickly retrieve the selected spatial entity and the attribute list, and then perform scientific statistics and computation according to the specific application. The data stored in Geographic Information System has multiple properties of the original data, which can be classified and extracted again according different requirements. For instance, spatial information may be shown in different ways such as color, line, symbol and annotation according to their different attributes so as to realize representation by thematic maps.

Digital terrain model analysis, or terrain analysis for short, is the most frequently used in constructional engineering. Terrain analysis refers to representation of topographic information in the digitized form, and consequently, it is used for automatic computation of topographic factor data such as gradient, slope direction and curved surface area [6]. For instance, terrain analysis can be used to extract all kinds of topographic factors automatically, to make terrain profiles, to divide earth surface morphotypes and so on. In engineering reconnaissance survey and design, it is often used for route selection of lines, site selection of buildings, etc. Terrain data may also be used for matching analysis of data from professional departments, such as land use planning study, thereby to realize data application in multiple fields.

D. Visualized representation of three-dimensional GIS. In traditional survey, the results of topographic maps are usually output in the form of paper maps or two-dimensional electronic maps in CASS software, thus two-dimensional visualized representation of surveying results were used. However, with the development of computer graphic technology and graphic processing technology, a kind of dynamic and interactive three-dimensional visualized products has appeared in GIS, which makes it possible for the geographic entities in the real world to be represented by using models of graphic and visual three-dimensional spatial graphs.

Three-dimensional GIS technology is the expansion and extension of GIS technology. After the information on $\mathrm{X}$ and $\mathrm{Y}$ axes are processed by two-dimensional GIS, the information on $\mathrm{Z}$ axis are projected onto a model so that all the information on $\mathrm{X}, \mathrm{Y}$ and $\mathrm{Z}$ are displayed, which can represent plane relationship and vertical relationship between spatial objects, and consequently, the geographic data are transformed into visual geographic information [7].

In addition, it is also possible to participate in the geographic information processing environment with the help of virtual reality technique, and through visual sense, hearing sense, tactile sense and body shape, gesture or password of a person. It gives the users even more authentic feelings, and thus an entirely fresh progress has been brought to the representation form of traditional surveying results [3].

\section{Development of Modern Surveying}

The superb working efficiency and digital accuracy of GIS technology has created economic benefits for engineering construction. The new achievements of modern science and technology should be effectively applied in survey teaching to provide new tools and means for surveying science. With the aid of GIS technology, engineering survey has realized automation of data acquisition and processing, intelligentization of surveying process control and system behavior, digitization of surveying results and products, visualization of surveying information management, 
and networking of information sharing and dissemination. Looking forward to the future, surveying science shall be developed from one-dimensional and two-dimensional into real-time three-dimensional and even four-dimensional, which shall be integrated into a multi-discipline and further strengthen graph, image, communication and data processing capabilities. The service of surveying shall be further broadened to play a more important role in such aspects as engineering design, supervision and assessment and in such fields as regional planning, transportation management, environmental protection and house property transaction. In combination with other related disciplines, it shall be used to develop various engineering thematic information systems and take part in decision making and management of projects [8].

\section{Summary}

Traditional surveying technologies are mostly based on manual measurement, which are rather complicated in collecting and processing geographic information resources and related data. Thus the low efficiency and less accurate surveying results cannot meet the requirements of actual surveying engineering. However, the application of GIS technology in surveying has effectively solved all the problems in traditional survey. Moreover, this technology can be extended and developed continuously on the basis of the original technology. With the improvement of our scientific and technological level, the innovation and development of GIS technology shall bring about even greater surprises to engineering survey.

\section{Acknowledgments}

The authors are grateful to the financial support of the Fund Program: 2014 Teaching and Research Project supported by Hebei Normal University of Science \& Technology (Project No.: JYYB201421).

\section{References}

[1] X.L. Luo and J.H. Zhang: Scientific and Technological Information (9), ( 2014), 17-18.

[2] D.R. Li, Y.J. Wang, Z.F. Shao: Wuhan University Journal (Information Science Edition) 1 (37), (2012), 1-5.

[3] L. Wu, et al.: Methods and Applications of Geographic Information System (Science Press, China 2001).

[4] S.N. Liu: New Surveying and Mapping Technology for Qingdao City Large-Scale Topographic Map (Ocean University of China, 2011).

[5] S. Yan: Urban Architecture, 18, (2013), 124-137.

[6] X.Y. Huang, J.S. Ma: Introduction to Geographic Information System (Higher Education Press, China 2008).

[7] L. Xiao, E. Zhong, J.Y. Liu, et al.: China Journal of Image and Graphics 9 (6), (2001), 842-848.

[8] J.S. Ning, J.Y. Chen, D.R. Li, et al.: Introduction to Surveying and Mapping (Wuhan University Press, China 2004). 